\title{
Los modelos de innovación: complejidad versus creatividad; el dilema de una simplicidad que genera resultados útilles
}

\author{
Innovation models: complexity versus creativity; the dilemma \\ of a simplicity that generates usefull results
}

Picado Vanegas, Armando; Editor Academico Dr. Angel Sol Sanchez

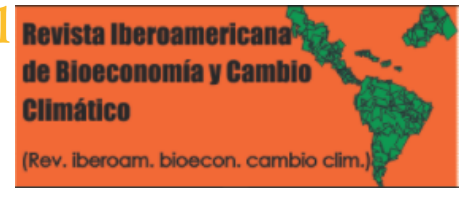

\author{
Armando Picado Vanegas \\ armanpicv2000@yahoo.com \\ UNAN - Managua, FAREM - Matagalpa, Nicaragua \\ Editor Academico Dr. Angel Sol Sanchez \\ Colegio de Postgraduados, Mexico, México
}

Revista Iberoamericana de Bioeconomía y Cambio Climático

Universidad Nacional Autónoma de Nicaragua, León, Nicaragua ISSN-e: 2410-7980

Periodicidad: Semestral

vol. 2, núm. 1, 2016

czuniga@ct.unanleon.edu.ni

Recepción: 15 Octubre 2015

Aprobación: 15 Febrero 2016

URL: http://portal.amelica.org/ameli/journal/394/3941750012/

DOI: https://doi.org/10.5377/ribcc.v2i1.5686

Autor de correspondencia: armanpicv2000@yahoo.com
Resumen: Para llevar a efecto procesos de innovación, prima la necesidad del cambio y la suficiente creatividad para llevarlo a cabo, de forma que se pueda inducir el desarrollo de un modelo adecuado a dichas necesidades y al entorno donde debe aplicarse, pero es evidente que aún existen obstáculos que no están permitiendo los enlaces adecuados entre el desarrollo de las tecnologías y la innovación del sector agropecuario. La verdad es que nuestros países, constantemente agobiados por problemas graves de inseguridad alimentaria, salud, hacinamiento, educación, exclusión social, degradación ambiental, migración, difícil acceso al agua y a la tierra, entre otros elementos que conforman la baja calidad de vida que nos afecta, la innovación es un proceso, cuya búsqueda, está basado en el encuentro de caminos viables para la sobrevivencia. La gestión de la innovación parte en nuestro caso desde la base productiva.

Palabras clave: Innovación, modelos, entorno, pertinencia.

Abstract: In order to process innovation effect, prime the need for change and creative enough to carry it out, so that it can induce the development of an appropriate model to these needs and environment where it should be applied, but obviously still exist obstacles are not allowing adequate links between the development of technology and innovation in the agricultural sector. The truth is that our countries constantly burdened by serious problems of food insecurity, health, overcrowding, education, social exclusion, environmental degradation, migration, difficult access to water and land, among other elements of the low quality of life that we it affects, innovation is a process, whose search is based on the meeting of viable ways for survival. Management innovation is in our case from the productive base.

Keywords: Innovation, models, environment, relevance.

\section{Notas DE AUTOR}




\section{INTRODUCCIÓN}

El presente ensayo trata de poner en perspectiva que en el contexto latinoamericano, es imposible encasillar los modelos de innovación, cuya complejidad proviene claramente de influencias lineales, por su estrecha relación con las ciencias duras, lo que invisibiliza a la creatividad cotidiana, donde innovar implica sobrevivir en un entorno donde prima más la necesidad de resolver la inmediatez del día a día, que la generación de excedentes para producir riqueza económica.

La innovación en su proceso, va aportando nuevos conocimientos y sus resultados tienen evidentemente una utilidad tangible. La disparidad en el desarrollo tecnológico actual entre los países del primer mundo, comparado con los países en vías de desarrollo, es abismal; ¿Cómo se debería abordar el tema de la innovación desde la visión del entorno socioeconómico latinoamericano? Y más específicamente; ¿Cómo abordar la innovación en entornos de pobreza, inseguridad alimentaria y las amenazas del gradual y latente cambio climático?

\section{LOS MODELOS}

Existen diferentes concepciones sobre la definición de la innovación y los modelos que subyacen en el contexto actual. En el documento del SBI1 (2008), se exponen tres modelos de innovación: "la ciencia empuja (1950-1965), el mercado jala (década de los 60) y un modelo sistémico de gestión de la innovación (1969-1985) (Delgado \& Escobar, 2009). "La innovación de acuerdo con Freeman (1974), se considera una actividad bilateral, aunque algunas teorias se centran sólo en enfoques unilaterales, ya se trate de "teorias de innovación basadas en el tirón de la demanda" y "teorias de la innovación basadas en el empujón de la ciencia". No obstante han surgido aportes que consideran ambos enfoques, conocidos como modelos interactivos de los procesos de innovación. En estos modelos se concede importancia a las retroalimentaciones entre diferentes fases del esquema e innovación y a las diferentes interacciones que relacionan las diversas fuentes de conocimiento a lo largo de las etapas del proceso innovativo" (Infante, Ortega, \& Ortiz, 2011).

"La postura tradicional de hacer investigación reconoce la existencia de una relación causal entre ciencia y tecnología que se representa mediante un modelo lineal que describe la génesis de una innovación tecnológica como un proceso secuencial que partiendo de la ciencia llega a un producto o proceso comercializable" (Morales \& Aguilar, 2002). Según (Pérez, 2005), haciendo un resumen descriptivo de los modelos indica que;" i) El Modelo Lineal: "Este modelo describe la innovación como un proceso de un único camino que empieza con la investigación básica, pasa por la investigación aplicada y el desarrollo tecnológico y acaba con el marketing y lanzamiento al mercado de la novedad"; ii) El Modelo de Marquis: "Este modelo reconoce que la idea de una innovación no se desprende necesariamente de la investigación como lo plantea el modelo lineal, sino que puede surgir de cualquier parte de la empresa"; iii) Modelo de Kline: "Propone un modelo de innovación que conecta la ciencia y la tecnología en todas sus etapas y no solo al comienzo" (Pérez, 2005).

En la mayor parte de los casos, es imposible encasillar una modelación de la innovación que no esté relacionada con la otra. Existen algunos esfuerzos por resumir la forma en que se podría concretar una síntesis teórica de los modelos de innovación. "Del análisis de las propuestas realizadas por distintos autores se deduce que existen algunos modelos sobre el proceso de innovación más extendidos y aceptados en la literatura general. Concretamente los modelos más destacados son los Modelos Lineales, Los Modelos por Etapas, los Modelos Interactivos o Mixtos, los Modelos Integrados y el Modelo en Red" (Velasco, Zamanillo, \& Gurutze, 2015). No obstante, siempre existe la tendencia práctica de que el proceso tiende a ser más liberal, diversificado y proclive a mezclarse de acuerdo con las necesidades que atiende, el contexto y la interacción que propicia en función de la búsqueda de resultados tangibles.

En una publicación del sistema boliviano de innovación (SBI) se hace alusión al modelo sistémico aduciendo que; "la innovación es más que el producto de la investigación y desarrollo aplicado a la resolución 
de problemas sociales y económicos. La manera en la que la innovación nace, crece y se difunde, depende de un conjunto de factores sociales, económicos y organizativos".

Entonces, es importante reconsiderar la relación Ciencia-Tecnología-Sociedad, no según una lógica lineal y unidireccional, sino circular y sistémica"(Delgado \& Escobar, 2009). Esta concepción de los modelos de innovación podría estar integrando más variables que las concepciones anteriores, principalmente en lo correspondiente a su configuración y podría considerarse un avance importante en la visión sobre la innovación en su mejor sentido.

\section{LA VISIÓN DE LOS MODELOS EN NICARAGUA}

Según FUNICA, "los antecedentes del desarrollo tecnológico inician el modelo centralizado de los años 60 y 70 donde la "mayoría de los paises en la región siguió el esfuerzo de construir sus propias capacidades de investigación y transferencia de tecnologia, basadas en las recomendaciones de la CEPAL y a partir de las experiencias en paises desarrollados. De aqui surgen los Institutos Nacionales de Tecnologia (INIAS o INTAS) que fueron durante esa década y la siguiente los principales centros de referencia tecnológica en cada país, bajo la responsabilidad de los Ministerios de Agricultura. Según la misma fuente, en su descripción del modelo de las décadas 80-90 aduce que; "A finales de los 70 se visualizaba que cada vez era menos efectivo continuar con un modelo que ya no respondia a las exigencias que se habian presentado al final de la década. Por lo que un nuevo esquema se impulsa. El paradigma de este modelo fue incorporar las experiencias de iniciativas de actores diferentes que trabajaban en opciones alternativas". En términos más actuales implican que; "A partir del 2000 y hasta el presente, Este nuevo esquema se visualiza en modelos integrales de investigación-asesoria-educación, asícomo en la utilización de metodologias participativas, que interrelacionan más efectivamente a los actores principales de la innovación tecnológica (agricultores, extensionistas e investigadores)con otros actores de la cadena (financiadores, proveedores de insumos, transformadores y comercializadores)" (FUNICA, 2009).

En el año 2010 se realizó en Nicaragua, un evento promovido por la cooperación suiza, donde participaron representantes de países como; Bolivia, Cuba y Nicaragua. En sus reflexiones se muestra que el modelo de innovación tiene como aspectos positivos que deben tomarse en cuenta como; la demanda, articulación investigación formal y conocimiento local, la investigación aplicada, el uso de tecnologías pertinentes y eficaces y la gestión de conocimiento. También exploraron algunos aspectos negativos como; el alcance reducido de las innovaciones, el tipo de modelo de innovación dominante, la configuración de actores públicos de arriba hacia abajo, la actual visión de oferta antes que atender a demanda. Concluyeron que se debe profundizar en los Sistemas Nacionales anclados en lo local para facilitar la articulación del conocimiento científico y el saber campesino (en dos vías). (COSUDE, 2010)

\section{COMPLEJIDAD VERSUS CREATIVIDAD}

El avance de las tecnologías y sus subsecuentes innovaciones en todas las áreas de la ciencia han generado un sinnúmero de opciones de adopción de conocimientos, artefactos, medios y técnicas, cuya utilidad muchas veces está más circunscrita al ámbito, territorio, población y contexto socioeconómico donde han sido creadas. Muchas veces, esas tecnologías e innovaciones, serán objeto de un proceso de re-innovación adaptativa que permita a los usuarios aprovechar sus utilidades en un entorno distinto al que fueron creadas. "La investigación en agricultura ha tomado un curso básicamente tecnológico innovativo y los esfuerzos están dirigidos a fomentar la participación de la empresa privada dentro de este proceso, siguiendo un modelo no lineal en el que los actores sociales y el territorio han logrado un espacio en la definición de las características y el funcionamiento de la cadena productiva en busca de desarrollo rural"(Morales \& Aguilar, 2002). 
Según (Pedroza, 2010) "Para desarrollar una agricultura con conocimiento para la innovación tecnológica es necesario la aplicación de los siete enfoques contemporáneos de investigación, bases para desarrollar un modelo sistémico de innovación tecnológica del agro nicaragüense. Estos enfoques son: i) Enfoque de Cadenas Productivas: Modelo I \& D; ii) Enfoque Sistémico: Redes; Pensamiento Sistémico; SI PSひE; C I P P. iii) Enfoque de Competitividad: Entorno: Mercados; Clientes; Demanda... FONTAGRO, FAITAN, Red SICTA, etc. iv) Enfoque Integrado de Investigación y Extensión en Sistemas Agropecuarios (IESA). v) Enfoque de Desarrollo Rural Incluyente: Género, Participativo, Legitimidad, Compromiso, DEL; Territorialidad; Consistencia $y$ Capacitación de Clientes, Beneficiarios, Socios, Usuarios. vi) Enfoque de Politica Tecnológica. vii) Enfoque de Conservación y Manejo Sostenible de los Recursos Naturales"(Pedroza, 2010). Hacer una confluencia de estos enfoque y tomarlos como punto de partida es importante, pero su complejidad debe ser abordada con conocimiento, por lo que es determinante convertirlos, en una política pública de Desarrollo Rural Territorial Sustentable que aplicada a los actores,consiga en términos prácticos, los resultados que se esperan de un proceso de innovación incluyente, comprendido, aplicado y aprovechado, por todos los interesados aun si estos no forman parte de la academia o del sistema que lidera el proceso.

Tomando como referencia la realidad rural latinoamericana y las dificultades que impone el medio ambiente, el limitado acceso a recursos y la amplia brecha tecnológica que afecta directamente las bases productivas agrarias, el acceso alimentario y los ingresos, debe hacerse visible que éste contexto facilita la generación de muchas innovaciones, casi imperceptibles. "Como contraparte a la generalizada ausencia de innovaciones radicales, la poca inversión en I $\mho D$ y la naturaleza más bien receptora de un sector más bien rezagado como el agropecuario; cobran vital importancia las "innovaciones menores" como determinantes en la medición. Es alli, en el estudio de esas pequeñas cosas que suceden en el campo, como se puede entender con mayor precisión lo que sucede en el sector agropecuario en cuanto a innovación" (Saavedra, y otros, 2011)

Es importante procurar la gestión del conocimiento partiendo desde los medios ambientes más básicos a fin de favorecer la creación de capital social local. "En la actual era del conocimiento, existe evidencia de que el desarrollo económico y social de una región determinada o de un sector como el agrícola, en particular, está basado en la formación de capital humano, en la investigación que impulsa la generación de conocimiento, en el desarrollo y en la transferencia tecnológica" (Rendón, 2010). Es por ello que en algunos países latinoamericanos tomando en cuenta en principio, su propia pertinencia cultural, se han logrado avances significativos en la gestión del conocimiento. "Cuando se incluyen los criterios de los productores y productoras en el diagnóstico de los problemas y selección de las soluciones a experimentar para la investigación adaptativa en las fincas con los Comités de Investigación Agricola Local (CIAL) se encuentra un aumento en el número y diversidad de innovaciones productivas en los sistemas de producción"(Cambio Andino, 2010).

\section{LA CREACIÓN DE UN AMBIENTE INNOVADOR}

El acceso a las innovaciones desde la perspectiva de sistemas, requiere de la participación activa de todos los sectores vinculados al desarrollo del territorio, por lo que es importante crear condiciones idóneas que cimenten una estructura social que haga posible la interacción amplia para facilitar los flujos de comunicación y aprovechamiento del conocimiento. "La innovación requiere de un ambiente o cultura organizativa que la promueva y la favorezca. Una cultura organizativa sustentada en valores como flexibilidad, asunción de riesgos, compartir, dinamismo, sugerir, entusiasmo, inquietud, creatividad, etc., hace que el proceso de innovación tenga lugar de forma más ágil y dinámica"(Velasco, Zamanillo, \& Gurutze, 2015).

A nivel de adopción, es determinante la influencia del tejido social que involucra a los actores de base. "Los efectos de los vinculos del productor con diversos actores relevantes para sus decisiones sobre el volumen o intensidad de adopción. Se pone atención particular a la interacción del productor con los agentes externos (e.g.,vendedores de insumos, compradores de producto, extensionistas, etc.) que son ampliamente reconocidos como actores clave en la literatura sobre difusión de innovaciones" (Monge \& Hartwich, 2008). 


\section{PROPUESTAS PARA EL DESARROLLO DE LA INNOVACIÓN AGROPECUARIA EN NUESTRO CONTEXTO}

"Como alternativas al discurso desarrollista, la innovación social rescata y valora las variadas formas que asumen las realidades sociales y politicas del mundo desde la experiencia de los actores vulnerables y sus estrategias en la vida cotidiana y para resolver por ellos mismos, problemas dificiles de existencia. Esto identificó la potencialidad de procesos participativos de innovación y de coproducción de conocimiento que resultaba de alianzas politicas novedosas"

(IEP, 2013). El proceso de búsqueda de alternativas o nuevos modelos endógenos de innovación es determinante para incentivar la participación de los actores más relevantes.

En principio deben reconocerse, las limitaciones que plantea la ruralidad latinoamericana, y en perspectiva, la realidad de la innovación agropecuaria en Nicaragua. "Urge construir mistica y compromiso interinstitucional e imaginar modelos de gestión que puedan manejar la complejidad. Interesa, pues, alejarse gradualmente del paradigma mecanicista, verticalista, lineal, deductivo, dependiente"(IICA, 2009). Se reconoce entonces que el modelo actual requiere de un golpe de timón en un nuevo contexto donde según el IICA, "viejosproblemas se agregan, se debilita la seguridad alimentaria, los problemas del hambre, la ruptura de los tejidos sociales, los bajos niveles de productividad, el envejecimiento de la población rural, entre otros"(IICA, 2009). Estos son elementos a tomar en cuenta para impulsar procesos de innovación acordes a la realidad objetiva del sector rural en Latinoamérica.

El conocimiento del tejido social que involucra a los actores relevantes y claves de la innovación es básico para comprender sus interacciones y las vías que recorren para la búsqueda de información. "En efecto, al mapear un sistema producto cualquiera con el propósito de comprender la trayectoria que sigue el intercambio de conocimientos e información entre los diversos actores, invariablemente se descubre una mayor o menor densidad de interacciones entre agricultores, destacando el hecho de que un número relativamente reducido de actores aglutina varias redes pequeñas en otras más grandes y actúa como un poderoso catalizador de la innovación en toda una región, sistema producto o sector"(SAGARPA; FAO., 2006).

Uno de los factores más esenciales y determinantes en el replanteamiento de las innovaciones conjuga la misma interacción del hombre con su medioambiente. "El manejo sostenible de los recursos y la revitalización del sector agropecuario son herramientas que permitirian elevar la calidad de vida de las poblaciones más vulnerables y atenuar los efectos de la desertificación y el cambio climático"(MASHAV; CINADCO, 2015). El futuro de la agricultura industrial y familiar depende no solamente de la actual capacidad de innovar, sino de la habilidad de prever y proveer suficientes recursos alimentarios para el presente y el futuro.

\section{CONCLUSIONES}

- Los analistas de la innovación plantean la importancia de cambios que induzcan a la mejora en los resultados que generan las innovaciones. Los aspectos más relevantes que dificultan la aplicación de procesos más inclusivos están referidos a la amplia brecha que existe entre los actores encargados de canalizar las innovaciones y los usuarios finales, el acceso limitado que obtienen los protagonistas y la ausencia de pertinencia de algunas innovaciones vinculadas principalmente, con la cultura productiva y la realidad socioeconómica del tejido social productivo latinoamericano y nicaragüense.

- El hecho es, que la innovación es un concepto dinámico y no se conoce un modelo universal que aglutine las diferentes corrientes de pensamiento acerca de su enfoque, en particular por que la innovación es multidimensional y diversa en su aplicación. Es importante tomar en cuenta que "la prospección", vista como estudio del área de innovación en sus características más visibles y como una herramienta para obtener conocimiento, puede ser una vía para entender la forma en que gestiona y se producen los cambios en el ámbito agropecuario. Con respecto a éste aspecto y poniendo en perspectiva a la innovación en 
Nicaragua, (Saavedra, 2011) refiere; "En nuestro pais el tema de prospección se ha realizado de manera dispersa, segmentada y no ordenada, hay muchos estudios de cadenas, de brechas tecnológicas, sin embargo, estos no han sido desarrollados a partir de una agenda consensuada de los actores de las cadenas"(Saavedra D. , 2011). En este sentido, urge disponer procesos ordenados que permitan conocer la innovación desde una prospección participativa, que ayude a ordenar el conocimiento y disponerlo a la orden delos actores interesados.

- En principio es importante retomar algunas herramientas, cuyos resultados pueden ser útiles para aplicar o ensayar procesos inmediatos para el mapeo y la puesta en marcha de algunas innovaciones. "Los estudios de caso permitirian abordar otro aspecto importante, cual es el de la multiplicidad de relaciones (redes) que ligan a un mismo conjunto de actores (p.ej., relaciones de intercambio de productos, de información, de acceso a crédito, etc.)"(Monge \& Hartwich, 2008). Otra vía es el uso de herramientas tecnológicas a corto plazo como las tecnologías de la información y la comunicación. "La apropiación social de las TIC se logra cuando convergen en una comunidad las necesidades de comunicación, las culturas de colaboración y el interés por construir una identidad local en contextos digitales y de globalización"(IDI; USACH, 2010).

- En términos de "seguridad medioambiental" debe existir mayor prospección sobre los efectos positivos o negativos de las innovaciones sobre el medioambiente y las implicaciones que se promueven a corto, mediano y largo plazo, de forma que una de las variables determinantes en la aplicación y difusión de una innovación, sea el efecto de la misma, sobre los actuales y potenciales recursos naturales. La problemática del agua y la vulnerabilidad en la seguridad alimentaria, son factores determinantes para la garantía del futuro de las nuevas generaciones, de la dinámica ambiental de los territorios y la sobrevivencia global. Es probable que las nuevas innovaciones, impliquen que aprendamos a almacenar alimentos para plazos muy largos, con el fin de guardarlos en el tiempo de abundancia, para garantizar la alimentación en tiempos de crisis, en una imitación moderna de; según el antiguo testamento de la biblia (el pueblo egipcio, almacenando su producción,para tener comida en los años en que la naturaleza no permitirá acceder a los alimentos).

- La innovación en el contexto de Nicaragua y en la mayor parte de los pueblos, cuya cultura agraria carece de las sofisticadas atribuciones de la agricultura industrial, los modelos de innovación implican directamente procesos de mejora productiva donde la complejidad es confrontada por la creatividad de los usuarios protagonistas para adaptar las tecnologías a las que se logra acceder, a las acondiciones locales donde se resuelve el dilema de la generación de resultados útiles en la simplicidad que requiere la inmediatez para acceder a recursos productivos que permiten la diaria sobrevivencia.

\section{Literatura Citada}

Cambio Andino (2010). Metodologías Participativas para la Innovación Agrícola. Líma, Perú: Cambio Andino.

COSUDE (2010). Memoria: Talller de Intercambio de Experiencias en Innovación y Tecnología Agropecuaria. Managua, Nicaragua: Cooperación Suiza para el Desarrollo.

Delgado, F., \& Escobar, C. (2009). Innovación Tecnológica, Soberanía y Seguridad Alimentaria. La Paz, Bolivia.: AGRUCU-CAPTURED. / Plural Editores. 2009.

FUNICA (2009). Innovación Tecnológica: Nuevos retos para desarrollar alianzas público-privadas en Centroamérica. Managua, Nicaragua: RUTA.

IDI; USACH (2010). Modelos Cooperativos para el Acceso a Internet en Sectores Rurales. La experiencia de COOPESIC y sus aprendizajes. Santiago de Chile: Fundación para la Innovación Agraria.

IEP. (2013). Escalando las Innovaciones Rurales. Lima, Perú: Instituto de Estudios Peruanos.

IICA. (2009). Innovaciones rurales y tecnológicas en el nuevo modelo de desarrollo. Cadenas agroalimentarias: Innovaciones sociales y tecnológicas en el nuevo modelo de desarrollo en los territorios rurales. COMUNIICA, 6-24. 
Infante, Z., Ortega, P., \& Ortíz, C. (2011). Estrategias de Innovación y Transferencia de Tecnología Agrícola Orgánica en la Localidad de Los Reyes, Michoacán, México. Michuacán, México.: Universidad Michuacana de San Nicolás de Hiidalgo .

MASHAV; CINADCO (2015). Planificación, Gestión e Innovación sel Sector Agrícola. Tel Aviv, Israel.: Agencia Israelí de Cooperación Internacional para El Desarrollo / Centro Internacional de Cooperación para El Desarrollo.

Monge, M., \& Hartwich, F. (2008). Análisis de Redes Sociales aplicado al estudio de los procesos de innovación agrícola. REDES, 1-31.

Morales, J., \& Aguilar, C. (2002). La investigación de sistemas agropecuarios en Latinoamérica. Modelo descriptivo. ALPA, 184-192.

Pedroza, M. E. (2010). Un Nuevo Modelo de Innovación Tecnológica del Agro Nicaragüense. Encuentro, 78-81.

Pérez, C. (2005). Modelo de Innovación Tecnológica Basado en Enfoque de Redes Sociotécnicas: Estudio de Caso, Montana. Bogotá, Colombia.: Universidad de Los Andes.

Rendón, E. (2010). La Gestión Pública de la Innovación Agraria en el Perú: Antecedentes y perspectivas. Cuadernos de Investigación, EPG/ UPC, Escuela de Posgrado., 1-20.

Saavedra, D. (2011). La importancia de la prospección tecnológica en el sector agropecuario de Nicaragua. Managua, Nicaragua.: FUNICA.

Saavedra, D., Rugeles, L., Guaitero, B., Noreña, E., Arosa, C., \& Castillo, O. (2011). Innovación y modelos de organización empresarial en el sector agropecuario colombiano: Avances de un estudio. Bogotá, Colombia.: RAET.

SAGARPA; FAO. (2006). Análisis Prospectivo de Política de Ciencia, Tecnología e Innovación Agrícola. México: Secretaría de Agricultura, Ganadería, Desarrollo Rural, Pesca y Alimentación / Fondo de las Naciones Unidas para la Alimentación.

Velasco, E., Zamanillo, I., \& Gurutze, M. (2015). Evolución De Los Modelos Sobre El Proceso De Innovación. Dialnet. Obtenido de http/www.DialnetEvolucionDeLosModelosSobreElProcesoDeInnovacion-2499438\%20(1).pdf .Consultado el 24 Enero 2015. 